\title{
Risco de câncer associado ao uso de antidepressivos
}

\author{
Risk of cancer associated with the use of antidepressants
}

\author{
Camila Silva Bôaventura ${ }^{1}$, Andréia Nunes Guimarães ${ }^{1}$, Guilherme Rebello Soares ${ }^{1}$, Anna Milena \\ Barreto Ferreira Fraga ${ }^{1}$, Flávia Branco Cerqueira Serra Neves ${ }^{1}$, Milena Pereira Pondé ${ }^{2}$
}

1 Acadêmicos de Medicina, Escola Bahiana de Medicina e Saúde Pública (EBMSP), Salvador, BA. ${ }^{2}$ Médica psiquiatra. Professora adjunta, Disciplina de Farmacologia, EBMSP, Salvador, BA.

\section{Resumo}

Introdução: Alguns estudos sugerem que o uso de antidepressivos poderia aumentar o risco de câncer. Este estudo visa realizar uma revisão sobre o tema.

Método: Foi feita uma busca nas bases de dados MEDLINE e LILACS, utilizando como palavras de busca antidepressant, cancer e nomes das diferentes drogas antidepressivas.

Resultados: Onze artigos foram selecionados. Foram encontrados seis artigos sugerindo uma associação positiva fraca entre o uso de antidepressivos e o crescimento tumoral e cinco artigos que não sugeriam a associação.

Discussão: Os resultados dos estudos com relação ao risco de câncer associado ao uso de antidepressivos são ainda conflitantes. Na maioria dos estudos, a análise multivariada não mostra associação positiva em uso de antidepressivos e câncer, a não ser em casos específicos, como linfoma de Hodgkin.

Descritores: Antidepressivos, câncer, risco.

\begin{abstract}
Introduction: Some studies suggest that the use of antidepressants could increase the risk of cancer. This study aims at performing a review on this subject.

Method: A search was performed in the MEDLINE and LILACS databases using the keywords antidepressant, cancer and names of varied antidepressant drugs.

Results: Eleven articles were selected. Six articles were found suggesting a positive weak association between use of antidepressants and tumoral growth. In five articles this association was not found.

Discussion: The results of studies on increased risk of cancer associated with antidepressants are still conflicting. In most studies the multivariate analysis did not show positive association between use of antidepressants and cancer, unless in specific cases, such as Hodgkin's lymphoma.

Keywords: Antidepressants, cancer, risk.
\end{abstract}

\section{Introdução}

Existem relatos controversos na literatura a respeito do uso de antidepressivos (AD) e sua associação com o câncer (CA). Alguns autores sugerem uma associação entre o uso de $\mathrm{AD}$ e um aumento do risco de $\mathrm{CA}^{1-10}$. O CA é uma doença potencialmente fatal, e o uso de $\mathrm{AD}$ vem aumentando ao longo dos anos ${ }^{1,2}$, o que torna essa possível associação um tema importante de discussão. Cabe salientar, também, que os AD atuam no combate à depressão em pacientes com $\mathrm{CA}^{11-14}$.

Os autores que sugerem que o uso de $\mathrm{AD}$ diminui o risco de $\mathrm{CA}^{4,11-18}$ se baseiam em distintos argumentos. Em primeiro lugar, está a idéia de que a depressão causa uma depleção das células natural killers (NK) ${ }^{19}$, e das células de vigilância antineoplásicas ${ }^{20}$,

Correspondência:

Camila S. Bôaventura, Rua Edístio Pondé, 301/1303, Stiep, CEP 41760310, Salvador, BA. E-mail: milasb2004@yahoo.com.br 
aumentando o risco de desenvolvimento de CA. Com o tratamento da depressão, as células NK voltariam aos padrões normais, diminuindo o risco de CA. Um outro mecanismo que fundamenta essa idéia é o bloqueio dose-reversível e voltagem-dependente dos canais hEag1 causado pela imipramina e pelo astemizol. Esses canais foram detectados em várias linhagens de células tumorais, influenciando a sua taxa de proliferação ${ }^{11,13}$. Outro canal bloqueado pela imipramina e pela desipramina é o SK3 presente nas células TE671 do meduloblastoma humano, que também influencia na divisão das células cancerígenas $^{12}$. Há ainda um estudo que indica o uso de clomipramina como adjuvante na quimioterapia do CA, pois potencializa o efeito da vincristina em leucemias refratárias a esse medicamento ${ }^{16}$.

Outros autores consideram que os AD podem aumentar o risco de $\mathrm{CA}^{6,21}$, apresentando como argumentos o aumento comprovado da incidência de CA de cólon em ratos tratados com desipramina, após a intoxicação por azoximetano, em relação aos controles $^{21}$; a diminuição reversível da atividade das células NK após o tratamento com desmetilimipramina ${ }^{22,23}$, que, como citado anteriormente, é uma célula de vigilância antitumoral; e a semelhança estrutural entre os AD tricíclicos (ADT), como a amitriptilina, e os inibidores seletivos da recaptação de serotonina (ISRS), como a fluoxetina, com o ligante do receptor histamínico regulador do crescimento, o N, N-dietil-2-[4-(fenimetil) fenoxi] etanamina $\mathrm{HCl}$, que, in vivo, estimula o crescimento tumoral ${ }^{24}$.

Diante das controvérsias da literatura, este estudo visa realizar uma revisão sobre a associação entre o uso de $\mathrm{AD}$ e a estimulação do aparecimento e/ou crescimento do CA.

\section{Método}

Foi feita uma pesquisa nos seguintes bancos de dados: MEDLINE, Cochrane Database of Systematic Reviews and Controlled Trials Register, Update Software e LILACS. As palavras de busca foram: antidepressant, fluoxetine, paroxetine, imipramine, sertraline, nortriptiline, venlafaxine, citalopram, escitalopram, fluvoxamine, mirtazapine, trazadone, nefazodone, milnaciprane, duloxetine, maproptiline, amitriptyline, clomipramina e cancer. Foram incluídos apenas estudos com dados primários: estudos de coorte, caso-controle, caso-controle aninhado em coorte e relatos de caso realizados com humanos. Foram excluídos os artigos de revisão de literatura, metanálises, estudos em animais e aqueles que abordavam os AD no tratamento da depressão decorrente do CA.

\section{Resultados}

Considerando-se os critérios de inclusão e exclusão, foram selecionados 11 estudos publicados entre 1998 e 2005. Dos trabalhos selecionados, havia um com relatos de casos, oito estudos tipo caso-controle e dois estudos de coorte.

Três casos de CA de mama masculino ${ }^{25}$ foram associados ao uso de ISRS. Os ISRS foram fluoxetina, na dose de $20 \mathrm{mg} /$ dia durante 1 a 3 anos em dois casos, e paroxetina, na dose de $20 \mathrm{mg} /$ dia durante mais de 5 anos em um caso. Os autores sugerem que a avaliação da ação dos AD sobre o CA de mama em homens é mais simples de ser interpretada em relação às mulheres, devido ao menor número de fatores de risco que o gênero masculino apresenta em relação ao CA de mama. Além disso, a mama masculina sofre menos alterações fisiológicas ao longo da vida. Nos casos estudados, entretanto, os pacientes foram expostos a outras variáveis, provavelmente implicadas no surgimento de CA, como uso de outros medicamentos (por exemplo, a finasterida), consumo excessivo de álcool e história familiar de CA.

Foram incluídos oito estudos de caso-controle, sendo que em seis deles não foi evidenciada a existência de associação entre o uso de AD e o surgimento de CA, e em dois estudos a associação foi sugerida em situações específicas.

Dois estudos analisaram o risco de CA de ovário e o uso de AD. O primeiro deles analisou o risco de CA de ovário de acordo com o uso e o tipo de uso (regular ou irregular) de ADT, ISRS, fenotiazínicos e benzodiazepínicos $^{8}$. Foi considerado regular o uso em 4 dias por semana por pelo menos 4 semanas. Foram analisados questionários respondidos por pacientes atendidos durante 22 anos em quatro centros de saúde de importantes regiões metropolitanas americanas, obtendo-se uma amostra de 748 casos de mulheres portadoras de CA epitelial de ovário. Para cada caso, foram selecionados dois controles diagnosticados com algum tipo de CA e que não faziam uso das drogas e dois controles com outros diagnósticos, que não CA, e que também não utilizavam as drogas. Dos resultados, o que chama a atenção é o valor da odds ratio $(\mathrm{OR})=3,00(\mathrm{IC} 95 \%$ 0,80-10,50) para o uso regular de ISRS em pacientes com CA, comparados com os controles sem diagnóstico de CA. Quando foi feita a correção para outras variáveis, como menarca, menopausa, uso de contraceptivos orais, consumo de álcool e de tabaco, a OR caiu para 0,70 (IC95\% 0,101,10). Admitindo-se em conjunto as OR obtidas para o uso regular e irregular de ambas as classes de AD, em comparação com os dois grupos controles, não se obtém OR diferente de 1,00, sendo 0,20 o menor valor do intervalo de confiança e 3,30 o maior. Assim, os 
resultados não sugerem associação entre AD e CA de ovário.

O segundo estudo baseou-se na análise de um banco de dados de um órgão de vigilância em saúde, num período de 16 anos (1981-1997) ${ }^{3}$. Foram incluídos 314 casos de mulheres portadoras de CA epitelial de ovário, que foram pareados com 790 controles, mulheres entre 35 e 79 anos, com características sociodemográficas semelhantes aos casos e que não eram portadoras de CA. Foi analisado o uso de diversas classes de AD, através da sua prescrição durante um período de 6 meses e de seu uso contínuo ou não por mais de 6 meses. Os dados foram obtidos no banco de dados informatizado de farmácias ligadas ao órgão de saúde, que registram as informações referentes ao número de prescrições, às dosagens e à posologia de cada medicamento adquirido pelo paciente. Para os casos que possuíam apenas uma prescrição de $\mathrm{AD}$, num período de 6 meses, a OR foi de $1,1(0,69-1,90)$, e para aqueles com duas prescrições em 6 meses, de 0,71 (0,47-1,10). Em usuários nãocontínuos, por mais de 6 meses, a OR foi de $0,94(0,64-$ 1,4), e para usuários contínuos, por um período maior do que 6 meses, de $0,64(0,36-1,10)$. Os AD mais usados foram a doxepina, a amitriptilina e a imipramina. Nesse estudo, o fato de os ISRS passarem a ser amplamente utilizados apenas a partir da década de 80 prejudicou a análise de seu uso a longo prazo.

Um estudo de caso-controle ${ }^{6}$ indicou uma associação positiva, porém sem significância estatística, entre $\mathrm{AD}$ e CA de mama, com OR = 2,20 (0,8-6,30) para o uso de ISRS por 36 meses ou mais. Esse estudo baseou-se em banco de dados, previamente montado, de um estudo de caso-controle sobre CA de mama na Carolina do Norte, EUA, sendo que as informações sobre o uso de $\mathrm{AD}$ eram dadas pelos próprios pacientes. Foram selecionados 1.445 casos de mulheres com diagnóstico de CA de mama e que fizeram uso contínuo de AD por 3 ou mais meses antes do diagnóstico, pareados a 1.226 controles, mulheres com CA de mama e que não fizeram uso de $\mathrm{AD}$ antes do diagnóstico. $\mathrm{O}$ uso contínuo de AD foi relatado em 1/5 dos pacientes de ambos os casos e controles (após o diagnóstico de CA de mama), não sendo encontrada associação entre o aumento do risco de CA e o uso contínuo do AD (OR =1,00; IC95\% 0,7-1,20). Quando separados por classe de AD (ADT, ISRS, atípicos, lítio ou múltiplos tipos), todos os valores de OR foram inferiores a 1,10, sendo que o IC variou de 0,50 a 1,90 .

Outro estudo envolvendo o uso de AD e CA de mama, desenvolvido em Ontário, Canadá, analisou casos de CA relacionados no Registro de Câncer de Ontário e o uso de AD, levando em considerações variáveis sociodemográficas, reprodutivas, antropométricas, história clínica e psiquiátrica, estilo de vida e uso de outros medicamentos ${ }^{7}$. Selecionou-se 3.133 casos (mulheres com diagnóstico primário de CA de mama, com idade entre 25-74 anos no momento do diagnóstico) e 3.062 controles (randomicamente selecionados de uma população feminina de Ontário). Analisando-se o uso de todas as classes de AD e a prevalência total de CA, foi encontrada uma associação fraca, com OR = 1,17 (1,01$1,36)$, porém, quando esse valor foi corrigido considerando outros fatores de risco para CA, o resultado passou a não ser estatisticamente significante. Individualmente, o uso de sertralina e paroxetina sugere um fraco aumento no risco de CA de mama, com OR = $1,58(1,03-2,41)$ e $\mathrm{OR}=1,55(1,00-2,40)$, respectivamente. Na análise multivariável, entretanto, esse resultado perde sua significância, com valores de $\mathrm{OR}=1,45(0,88-2,40)$ para a sertralina e $\mathrm{OR}=1,60(0,93-$ $2,77)$ para a paroxetina. Esses resultados evidenciam a multiinfluência existente no desenvolvimento do CA de mama.

Contrariando os achados dos artigos anteriores, um estudo realizado com mulheres de 35 anos ou mais, na região de Saskatchewan, também no Canadá, sugeriu uma associação positiva entre o uso prolongado de ADT (11 a 15 anos) e risco de CA de mama ${ }^{26}$. Foram selecionados, nos bancos de dados do Saskatchewan Cancer Agency e do Saskatchewan Prescription Drug Plan, 5.882 casos de mulheres com CA de mama e 23.517 controles, escolhidos randomicamente da população sem antecedentes de CA, com uma média de quatro controles para cada caso. Do mesmo banco de dados, foram obtidas informações a respeito da prescrição de $\mathrm{AD}$, classe de droga prescrita, número de pílulas e dose da droga em cada pílula, porém não foram obtidas informações quanto à dose diária e quanto ao tempo de tratamento em cada prescrição. Levando em consideração todos os ADT, o estudo encontrou um aumento no risco de desenvolvimento de CA de mama em pacientes que fizeram uso dessas medicações por períodos entre $11 \mathrm{e}$ 15 anos, com risco relativo (RR) de = 2,02 (1,34 - 3,04). Baseado em estudos anteriores sobre a genotoxicidade dos ADT em Drosophila melanogaster, o autor divide os ADT em dois grupos: os genotóxicos - amoxapina, clomipramina, desipramina, doxepina, imipramina e trimipramina - e os não-genotóxicos - amitriptilina, maprotilina, nortriptilina e protriptilina -, para avaliar que drogas estariam relacionadas com o risco de desenvolvimento de CA de mama. Num período de uso entre 11 e 15 anos, os ADT genotóxicos causaram um aumento do risco de desenvolvimento de $\mathrm{CA}, \mathrm{com} \mathrm{RR}=$ $2,47(1,38-4,40)$, enquanto que o grupo dos nãogenotóxicos não foi relacionado ao aumento desse risco, com $\operatorname{RR}=1,92(0,93-3,95)$.

Um estudo desenvolvido pelo Food and Drug Administration (EUA) procurou determinar o risco de recorrência de tumores e o surgimento de um segundo tumor primário em pacientes que usaram $\mathrm{AD}^{10}$. $\mathrm{A}$ 
amostra foi retirada de um banco de dados que registra os casos diagnosticados de tumor, sua classificação, estadiamento, tratamento e acompanhamento. Os pacientes que estão nesse banco possuem benefícios quanto ao recebimento de medições, de modo que os autores utilizaram as informações da farmácia desse programa de saúde para selecionar os que usaram AD. Foram selecionados 1.467 pacientes que tiveram diagnóstico de CA de mama, de cólon ou melanoma maligno. Tais neoplasias foram selecionadas devido à sua prevalência, à sobrevida dos pacientes e à possibilidade de recorrência dos mesmos. Para cada caso, foi selecionado um controle pertencente ao banco de dados e com as mesmas características dos casos. Do total de pacientes com CA, 206 utilizaram AD, sendo que apenas 20 (4,2\%) tiveram recorrência e 17 (6,5\%) tiveram um segundo CA primário, uma amostra relativamente pequena quando comparada ao número inicial de pacientes com CA. Baseando-se nos valores de $\mathrm{OR}$ encontrados - relação entre $\mathrm{AD}$ e recorrências de $C A$, com OR =0,97 (0,52-1,78), e uso de AD e ocorrência de um segundo CA primário, com OR = 0,94 $(0,51-1,77)$-, o estudo sugere que não há relação entre os AD e o risco de recorrência e surgimento de um segundo tumor primário no período inferior a 6 meses.

No estudo realizado pelo Cancer Care Ontario, foram selecionadas mulheres com idade entre 25 e 74 anos, com diagnóstico de CA de mama primário, entre 1995 e 1996, nos Registros de Câncer de Ontário ${ }^{27}$. Os controles foram escolhidos randomicamente entre as mulheres de 25 a 74 anos no banco de dados do Ministério das Finanças de Ontário. Foram enviados para os casos e para os controles questionários autoaplicáveis que avaliavam: 1) dados sociodemográficos; 2) duração, dosagem e tipo de AD usado; e 3) potenciais confundidores. As mulheres que utilizaram AD por 2 semanas ou menos ou que usaram apenas nos últimos 6 meses antes do diagnóstico de CA foram excluídas da pesquisa. Foi observada uma alta proporção de história familiar e consumo de gordura nos casos, comparados aos controles. O uso de ADT, relacionado apenas à idade, teve fraca associação com o risco de CA de mama (OR = 1,2; IC95\% 0,8-1,8), porém sem significância estatística. Relacionando-se a idade e a OR dos diversos fatores, estimadas para o uso de ADT por 25 meses ou mais, houve um aumento no risco de CA de mama $(\mathrm{OR}=$ 2,5; IC95\% 1,2-5,1; multivariate odds ratio estimate $($ MVOR $)=2,1$; IC95\% 0,9-5,0). A paroxetina foi associada a um aumento do risco de CA de mama após o controle clínico da depressão (MVOR = 7,2; IC95\% 0,9-58,3). Já o uso de outros AD do tipo ISRS, como a amitriptilina e a fluoxetina, por mais de 2 anos, não foi associado a um risco aumentado de CA de mama (MVOR = 0,7; IC95\% 0,2-2,2).
Um importante estudo de coorte retrospectivo, realizado numa região da Dinamarca com $9 \%$ de toda a população, usou informações obtidas num banco de dados do Serviço Nacional de Saúde Dinamarquês, que reúne informações epidemiológicas das prescrições farmacêuticas cruzadas com informações do Centro Nacional de Câncer ${ }^{9}$. Foi selecionada uma população de 30.807 indivíduos que faziam uso de $\mathrm{AD}$ e se observou a freqüência de casos de CA, comparando-a com a freqüência esperada de acordo com variáveis como sexo, idade e época do ano. Foi avaliada também a relação dose/resposta na associação de AD e CA; para isso, a amostra foi dividida em grupos classificados como usuários ocasionais, periódicos ou regulares. Durante o estudo, foram encontrados 766 casos de CA, comparados a 746 esperados, resultando numa standardized incidence ratios $(\mathrm{SIR})=1,00(1,00-1,10)$ e refutando a hipótese de relação entre AD e risco de CA. Foi encontrada, porém, uma associação positiva entre o uso regular de ADT (cinco ou mais prescrições) e o surgimento de linfoma não-Hodgkin, com SIR = $2,50(1,40-4,20)$. Assim, os autores suspeitaram que o uso de ADT pode estar relacionado ao surgimento de linfoma não-Hodgkin ou, baseados na literatura, que a depressão estaria ligada à imunossupressão e conseqüente desenvolvimento de CA.

Haque et al. ${ }^{5}$ analisaram 635 pacientes com CA e usuários de $\mathrm{AD}$ com o objetivo de estabelecer o risco de crescimento tumoral pela utilização de paroxetina e outros AD. Os resultados indicam que o uso de paroxetina em relação a não-usuários de $\mathrm{AD}$ confere aumento que não é estatisticamente significante do risco de CA de mama em mulheres, com RR = 1,12 (0,961,31), porém não haveria relação com o tempo de uso. O uso exclusivo de paroxetina em relação a outros AD revelou uma $\mathrm{RR}=1,16(0,98-1,38)$, apresentando uma associação fraca e que não alcança significância estatística. O mesmo estudo não encontrou associação entre a utilização combinada de paroxetina e outros AD, $\mathrm{RR}=1,01$ (0,67-1,51).

Um estudo de caso-controle aninhado numa coorte ${ }^{1}$ analisou a associação entre AD e CA, especificamente ADT e ISRS e CA de mama. Foi utilizado como banco de dados o General Practice Research Database. Tratase de um banco de dados informatizado que contém informações a respeito de pacientes (dados socioeconômicos, consultas, internamentos, prontuários, exames e prescrições), colhidas por médicos de todo o Reino Unido, e que é especialmente utilizado para a realização de trabalhos científicos. Foram encontradas 3.708 pacientes como amostra. Como resultado, não foi observada relação entre o uso de AD (tanto ADT quanto ISRS) e o risco de CA de mama, com OR $=0,98(0,81-1,19)$ e $\mathrm{OR}=0,86(0,73-$ $1,00)$, respectivamente. 


\section{Discussão}

O uso de AD e o risco de desenvolvimento de CA envolvem alguns aspectos importantes. Um deles é a relação dessas drogas com o aumento de risco de CA, pois tal associação teria implicações clínicas, devido ao extensivo uso de $\mathrm{AD}$ e à falta de conhecimento público desse risco. Devem-se considerar, ainda, quais os $\mathrm{AD}$ que estariam envolvidos, quais os tipos de CA, qual a faixa etária mais afetada e qual o tempo de exposição à droga necessário para o desenvolvimento de CA. É importante salientar a necessidade de se buscar provas concretas da ausência de outros fatores de risco associados à neoplasia em indivíduos que fazem uso de medicações AD, para que se possa inferir a sua participação na promoção dessas enfermidades.

$\mathrm{O}$ relato de caso de Wallace et al. ${ }^{25}$ sugere a associação entre CA de mama masculino e o uso de ISRS. Tal hipótese é apoiada na idéia de que esse tipo de efeito é mais fácil de interpretar em homens do que em mulheres, à medida que a mama masculina sofre menos mudanças fisiológicas complexas que a feminina e, por isso, apresenta menos fatores de risco para o desenvolvimento de CA. Dentre os casos relatados, no entanto, havia a presença de outros fatores de risco, como o uso de finasterida, álcool e história familiar. O fato de existirem poucos estudos a respeito da utilização de ISRS por longos períodos justifica a seleção desse artigo. Porém, a análise de apenas três casos reduz a possibilidade de generalização desses dados.

Em estudo de caso-controle ${ }^{10}$, não houve associação positiva entre o uso de medicamentos AD e aumento da incidência de CA recorrentes ou tumores secundários. Esse estudo, entretanto, apresentou limitações como: pequeno número de pacientes nos grupos estudados, falta de correlação entre os casos de CA com um AD específico e definição arbitrária para se estabelecer o tempo que diferencia um tumor recorrente de um tumor primário resistente ao tratamento.

Cotterchio et al. ${ }^{27}$ obtiveram resultados que não sustentam a hipótese de que sempre o uso de qualquer AD está associado com o risco de surgimento de CA de mama. O estudo sugere que o uso de ADT e da paroxetina, por mais de 2 anos, pode estar associado a uma elevação do risco de surgimento de CA, porém, na análise multivariada, os resultados não alcançaram significância estatística. Os autores acreditam que não há evidências, até hoje, que sustentem uma mudança no uso freqüente de $\mathrm{AD}$, porém alertam que pode haver efeitos desconhecidos com o uso prolongado.

Coogan et al. ${ }^{8}$ concluem que não existe aumento do risco de CA epitelial de ovário após o uso de AD, ADT e ISRS. Esse estudo, entretanto, foi realizado com um pequeno número de usuários regulares de $\mathrm{AD}$, diminuindo, assim, a possibilidade de se avaliar o risco por duração, tempo do início do tratamento e envelhecimento com o uso do medicamento. Além disso, havia poucos usuários dos ISRS, pois essa classe de medicamento foi lançada no mercado apenas na década de 80 , sendo largamente utilizada a partir da década de 90. Outra limitação é que as informações sobre os pacientes, prévias ao diagnóstico, em relação ao uso de $\mathrm{AD}$, foram obtidas através de questionários aplicados por enfermeiras. Portanto, as informações obtidas quanto ao tipo de droga utilizada, tempo de uso e dose são diretamente dependentes da memória dos pacientes, podendo gerar um risco de viés.

Dublin et al. ${ }^{3}$ sugerem que não há evidência consistente sustentando a associação entre o aumento do risco de CA epitelial de ovário em mulheres e o uso de alguns tipos de AD. O fato de todas as informações coletadas sobre as características do uso dos AD serem obtidas dos dados da farmácia não descarta a possibilidade de os pacientes terem obtido os medicamentos em outras farmácias não cadastradas e não assegura que o paciente tenha aderido ao tratamento ou esteja fazendo uso correto das medicações.

Sharpe et al. ${ }^{26}$ associam o uso de ADT genotóxicos (amoxapina, clomipramina, desipramina, doxepina, imipramina e trimiprapina) com o aumento do risco de CA, posição esta não sugerida com o uso de ADT nãogenotóxicos (amitriptilina, maprotilina, nortriptilina e protriptilina). Essa idéia é sustentada após um período de uso superior a 10 anos. A limitação do estudo, entretanto, consiste na ausência de controle de outros possíveis agentes carcinogênicos que não os $\mathrm{AD}$.

O estudo de caso-controle de Moorman et al. ${ }^{6}$ não indicou o aumento do risco de desenvolvimento de CA de mama em usuário de AD. O único resultado encontrado com OR maior que a unidade não foi estatisticamente significante. No entanto, o estudo apresenta um resultado inesperado, até mesmo para os próprios autores, quanto ao carcinoma de mama in situ. As mulheres que faziam uso de AD tinham menos chance de desenvolver esse tipo de CA, quando comparadas com o grupo-controle. Nesse caso, os medicamentos teriam agido provavelmente como um fator de proteção contra o CA. A seu favor, pesa a grande amostragem de 1.445 casos, divididos entre carcinoma in situ e invasivo de mama.

Streingart et al. ${ }^{7}$ estudaram a relação de CA de mama com o uso de paroxetina e sertralina na avaliação através de modelo ajustado à idade. Os resultados encontrados não foram estatisticamente significantes nas análises multivariadas.

Os achados do estudo de caso-controle de Gónzalez-Pérez et al. ${ }^{1}$ não sustentam a hipótese de que o uso de AD aumenta o risco de desenvolvimento de CA de mama. Resultado encontrado mesmo quando se 
analisou diferentes variáveis, como: duração de uso do medicamento, dose diária ou tipo especifico de droga usada. Como cada médico fez a sua própria coleta de dados, algumas informações podem ter sido omitidas. Os dados não foram coletados visando ao estudo, já estavam nos prontuários.

No estudo de coorte realizado por Dalton et al. ${ }^{9}$, foi encontrada uma correlação entre o linfoma não-Hodgkin e o uso de AD. O estudo utiliza dados de um sistema que funciona de forma integrada na Dinamarca, no qual cada indivíduo possui um número nacional de identificação, e todas as farmácias da região estudada estão ligadas ao mesmo banco, reduzindo as perdas. Outro fator relevante é a utilização de todas as classes disponíveis de AD. Acredita-se que o linfoma não-Hodgkin seja fortemente associado a pacientes imunossuprimidos transplantados e pacientes com AIDS, mas nenhum dos casos se aplica ao estudo, pois as medicações para imunossupressão não foram administradas para os indivíduos em questão. A relação duração/resposta com AD para linfoma nãoHodgkin foi consistente em duas interpretações: na primeira, pesa o fato de a depressão estar associada à imunodepressão, além do que pacientes com depressão, severamente doentes, receberam muitas prescrições de AD; a segunda interpretação envolve indivíduos com tratamento a longo prazo com ADT e com etiologia positiva para CA, mas que não apresentam depressão. Todavia, limitando a interpretação dos resultados, o estudo não possui informações a respeito da dose diária de AD usada por cada indivíduo e da razão do uso desses medicamentos pelos pacientes.

O estudo realizado recentemente por Haque et al. ${ }^{5}$ sugere que não há nenhuma diferença entre o aumento do risco de CA de mama entre mulheres que usaram paroxetina e aquelas que utilizaram outros AD. Somando-se a isso, mulheres que usaram paroxetina por um período prolongado de 2 ou mais anos não apresentaram relação com o CA, quando comparadas com aquelas que usaram a medicação por um período mais curto. Porém, essa análise não foi ajustada através de uma comparação analítica com não-usuários de AD ou com informações associadas a outros fatores de risco existentes para CA de mama, como gestação e história familiar, nem levou em consideração as doses diárias utilizadas por cada paciente, o que poderia levar a uma redução dos valores de OR.

A maioria dos estudos analisados não encontra associação positiva entre o uso de AD e CA, ou a associação positiva não alcança significância estatística. Os relatos de caso sugerem uma associação entre CA de mama em homens e o uso de AD. A maioria dos estudos de caso-controle revisados não encontrou relação entre o uso de AD e o surgimento de diversos tipos de CA. Os estudos de coorte apresentaram divergência nos resultados sobre a associação entre os diversos tipos de CA e o uso de AD, uma vez que um deles encontrou relação entre o linfoma não-Hodgkin e ADT, enquanto que o de CA de mama apóia-se no mesmo resultado dos casos-controle, os quais não demonstram essa associação.

Concluindo, esta revisão bibliográfica indica resultados conflitantes quanto aos efeitos dos AD em promover, causar ou inibir o desenvolvimento dos diversos tipos de CA. É possível que os AD tenham um efeito adverso no crescimento de tumores cancerígenos apenas na presença de outros fatores carcinogênicos. Novos estudos na área são necessários para melhor definir a ação de cada AD como fator de risco ou de proteção em relação às várias formas de CA.

\section{Referências}

1. Gonzalez-Perez A, Garcia-Rodriguez LA. Breast cancer risk among users of antidepressant medications. Epidemiology. 2005;16(1):1015.

2. Lawlor DA, Juni P, Ebrahim S, Egger M. Systematic review of the epidemiologic and trial evidence of an association between antidepressant medication and breast cancer. J Clin Epidemiol. 2003;56(2):155-63.

3. Dublin S, Rossing MA, Heckbert S, Goff BA, Weiss NS. Risk of epithelial ovarian cancer in relation to use of antidepressants, benzodiazepines, and other centrally acting medications. Cancer Causes Control. 2002;13(1):35-45.

4. Abdul M, Logothetis CJ, Hoosein NM. Growth-inhibitory effects of serotonin uptake inhibitors on human prostate carcinoma cell lines. J Urol. 1995;154(1):247-50.

5. Haque R, Enger SM, Chen W, Petitti DB. Breast cancer risk in a large cohort of female antidepressant medication users. Cancer Lett. 2005;221(1):61-5.

6. Moorman PG, Grubber JM, Millikan RC, Newman B. Antidepressant medications and their association with invasive breast cancer and carcinoma in situ of the breast. Epidemiology. 2003;14(3):307-14.

7. Steingart A, Cotterchio M, Kreiger N, Sloan M. Antidepressant medication and breast cancer risk: a case control study. Int J Epidemiol. 2003;32(6):961-6.

8. Coogan PF, Rosenberg L, Palmer JR, Strom BL, Stolley PD, Zauber AG, et al. Risk of ovarian cancer according to use of antidepressants, phenothiazines and benzodiazepines (United States). Cancer Causes Control. 2000;11(9):839-45.

9. Dalton S, Johansen C, Mellemkjær L, Sørensen H, McLaughlin J, Olsen J, et al. Antidepressant medications and risk for cancer. Epidemiology. 2000;11(2):171-6.

10. Weiss SR, McFarland BH, Burkhart GA, Ho PT. Cancer recurrences and secondary primary cancers after use of antihistamines or antidepressants. Clin Pharmacol Ther. 1998;63(5):594-9.

11. Gavrilova-Ruch O, Schonherr K, Gessner G, Schonherr R, Klapperstuck T, Wohlrab W, et al. Effects of imipramine on ion channels and proliferation of IGR1 melanoma cells. J Membr Biol. 2002;188(2):137-49.

12. Carignani C, Corsi M. Inhibition of SK3 channels in the TE671 human medulloblastoma cell line by desipramine and imipramine. Eur J Pharmacol. 2002;448(2-3):139-42.

13. Garcia-Ferreiro RE, Kerschensteiner D, Major F, Monje F, Stuhmer W, Pardo LA. Mechanism of block of hEag1 K+ channels by imipramine and astemizole. J Gen Physiol. 2004;124(4):301-17.

14. Daley E, Wilkie D, Loesch A, Hargreaves IP, Kendall DA, Pilkington $\mathrm{GJ}$, et al. Chlorimipramine: a novel anticancer agent with a mitochondrial target. Biochem Biophys Res Commun. 2005;328(2):623-32.

15. Xia Z, Bergstrand A, DePierre JW, Nassberger L. The antidepressants imipramine, clomipramine, and citalopram induce apoptosis in human 
acute myeloid leukemia HL-60 cells via caspase-3 activation. J Biochem Mol Toxicol. 1999;13(6):338-47.

16. Tsuruo T, Iida H, Nojiri M, Tsukagoshi S, Sakurai Y. Potentiation of chemotherapeutic effect of vincristine in vincristine resistant tumor bearing mice by calmodulin inhibitor clomipramine. J Pharmacobiodyn. 1983;6(2):145-7.

17. Sauter C. Cytostatic activity of commonly used tricyclic antidepressants. Oncology. 1989;46(3):155-7.

18. Tutton PJ, Barkla DH. Influence of inhibitors of serotonin uptake on intestinal epithelium and colorectal carcinomas. Br J Cancer. 1982;46(2):260-5.

19. Frank MG, Hendricks SE, Burke WJ, Johnson DR. Clinical response augments NK cell activity independent of treatment modality: a randomized double-blind placebo controlled antidepressant trial. Psychol Med. 2004;34(3):491-8.

20. Whiteside TL, Herberman RB. The role of natural killer cells in immune surveillance of cancer. Curr Opin Immunol. 1995;7(5) 70410.

21. Iishi H, Tatsuta $M$, Baba $M$, Taniguchi H. Enhancement by the tricyclic antidepressant, desipramine, of experimental carcinogenesis in rat colon induced by azoxymethane. Carcinogenesis. 1993;14(9):1837-40.

22. Miller AH, Asnis GM, van Praag HM, Norin AJ. Influence of desmethylimipramine on natural killer cell activity. Psychiatry Res. 1986;19(1):9-15.

23. Eisen JN, Irwin J, Quay J, Livnat S. The effect of antidepressants on immune function in mice. Biol Psychiatry. 1989;26(8):805-17.

24. Brandes LJ, Arron RJ, Bogdanovic RP, Tong J, Zaborniak CL, Hogg $\mathrm{GR}$, et al. Stimulation of malignant growth in rodents by antidepressant drugs at clinically relevant doses. Cancer Res. 1992;52(13):3796-800.

25. Wallace WA, Balsitis M, Harrison BJ. Male breast neoplasia in association with selective serotonin re-uptake inhibitor therapy: a report of three cases. Eur J Surg Oncol. 2001;27(4):429-31.

26. Sharpe CR, Collet JP, Belzile E, Hanley JA, Boivin JF. The effects of tricyclic antidepressants on breast cancer risk. Br J Cancer. 2002;86(1):92-7.

27. Cotterchio M, Kreiger N, Darlington G, Steingart A. Antidepressant medication use and breast cancer risk. Am J Epidemiol. 2000;151(10):951-7. 